\title{
LEUKOENCEPHALOMALACIA IN A TWO-YEAR-OLD THOROUGHBRED COLT
}

\author{
A. ANOUSHEPOUR ${ }^{1}$, M. SAKHA ${ }^{1}$, S. OZMAIE $^{1}$, P. MORTAZAVI $^{2}$ \\ \& P. MOTTAGHIAN ${ }^{3}$ \\ ${ }^{1}$ Department of Clinical Science, Science and Research Branch, Islamic Azad \\ University, Tehran, Iran; ${ }^{2}$ Department of Pathobiology, Science and Research \\ Branch, Islamic Azad University, Tehran, Iran; ${ }^{3}$ Faculty of Veterinary Medicine, \\ University of Tehran, Tehran, Iran
}

\section{Summary}

Anoushepour, A., M. Sakha, S. Ozmaie, P. Mortazavi \& P. Mottaghian, 2020. Leukoencephalomalacia in a two-year-old Thoroughbred colt. Bulg. J. Vet. Med., 23, No 1, 138-142.

Leukoencephalomalacia is a disease of horses and donkeys caused by the ingestion of the mycotoxin fumonisin produced by the fungus $F$. proliferatum. In June 2015, a 2-year-old Thoroughbred colt kept in a feedlot around Tehran, Iran was presented with severe neurologic signs for clinical examination. Based on clinical evidences, history of ingestion of inappropriately stored commercially prepared feedlot concentrates and post mortem examinations, equine leukoencephalomalacia was suspected. Histopathologic findings, the results of feed fungal culture and mycotoxin analysis confirmed the disease.

Key words: fumonisin, leukoencephalomalacia, neurotoxic syndrome, Thoroughbred colt

Leukoencephalomalacia caused by ingestion of fumonisin toxins is seen worldwide, with most cases occurring in winter and early spring. Equine leukoencephalomalacia (ELEM) is also known as moldy corn poisoning, blind staggers, corn stalk disease and mycotoxic encephalomalacia. Typically there is an acute onset of signs 3 to 4 weeks after daily ingestion of contaminated feed. In some cases, the feed source is elusive, and the disease has been recognised with commercial feeds as well as corn (Raoofi et al., 2003; Robinson,
2003). There is no breed or gender predisposition, but usually it does not affect horses under 1 year of age. Amongst the domestic animals, horses are the most sensitive to fumonisin intoxication, the toxic effects of fumonisins in this species being dose-dependent. Fumonisin toxins (B1, B2, and B3), produced by Fusarium verticillioides and $F$. proliferatum (previously combined as $F$. moniliforme), interfere with sphingolipid metabolism, disrupting endothelial cell walls and basement membranes. Although all three are 
toxic, fumonisin B1 is the most common (Robinson, 2003; Reed, 2004; Smith, 2007).

Two clinical syndromes are associated to fumonisins intoxication. More common is the classic neurotoxic syndrome, but hepatotoxicosis also occurs in some horses (Reed, 2004; Gianntti et al., 2011). The neurologic syndrome includes progression from anorexia and depression to ataxia, circling, apparent blindness, headpressing, hyperesthesia, agitation, delirium, recumbency, seizures, and death. An early and consistent sign in affected horses is reduced proprioception of the tongue (Robinson, 2003; Reed, 2004; Smith, 2007).

ELEM has no unique findings on routine blood analysis. Serum liver enzymes and bilirubin are often high. Anaemia, leukocytosis, and leukopaenia have been reported. Markedly high protein concentration (i.e., $>100 \mathrm{mg} / \mathrm{dL}$ ) with normal nucleated cell count typifies cerebrospinal fluid (CSF) analysis of affected horses. Albumin, IgG concentrations and albumin quotients have been reported to be elevated (Smith, 2007).
In this report, a case of equine leukoencephalomalacia in a Thoroughbred colt in Iran confirmed based on histopathologic findings and detection of toxic concentrations of fumonisins in feed source is described.

\section{Case description}

In June 2015, a two year old colt kept in a feedlot around Tehran was presented for clinical examination. In addition to anorexia, apprehension, lethargy, somnolence, weakness, recumbency and muscle fasciculations as general clinical signs, the colt exhibited a variety of neurological signs such as ataxia, head pressing, circling and leaning to one side. The heart rate was mildly elevated and temperature and respiratory rate were normal. Signs of dehydration and self-trauma subsequent to ataxia and falling were apparent (Fig. 1). Blood samples were collected for complete blood count (CBC) and serum biochemistry. $\mathrm{CBC}$ revealed slight increase in PCV and neutrophilic leukocytosis. Serum AST, GGT and total bilirubin. were mildly elevated $(670 \mathrm{U} / \mathrm{L}, 137 \mathrm{U} / \mathrm{L}$ and $3.2 \mathrm{mg} / \mathrm{dL}$ ).

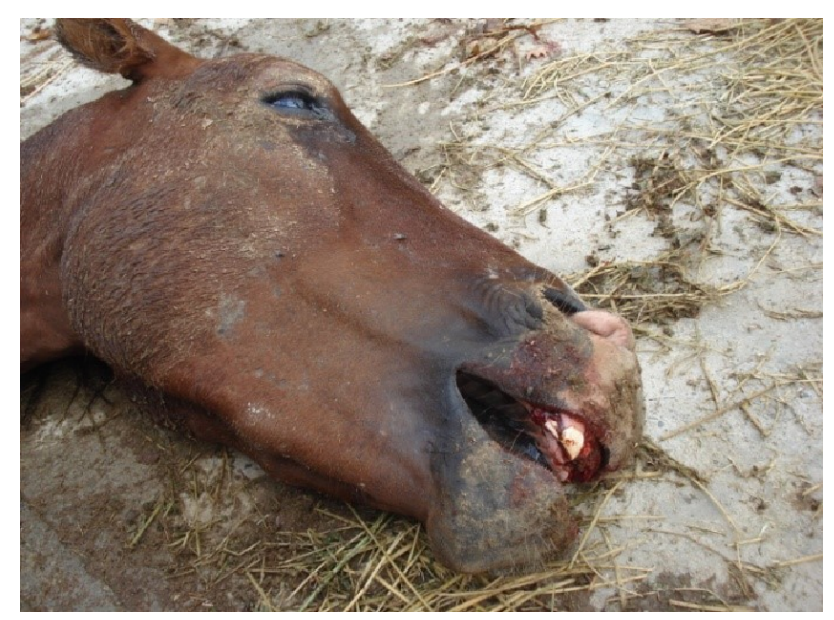

Fig. 1. Severe dehydration and self-trauma subsequent to ataxia and falling. 
Cerebrospinal fluid was collected from the lumbosacral space. CSF analysis showed increased protein concentration (98 mg/dL). Polymorphonuclear (PMN) counts were in normal ranges.

Based on clinical evidences, history of ingestion of inappropriately stored commercially prepared feedlot concentrates, equine leukoencephalomalacia was suspected. Because of severity of signs and poor prognosis, the colt was humanely euthanised.

At necropsy, no gross pathologic findings were seen in internal organs. Macroscopically, the brain was congested, having haemorrhages and friable consistency (Fig. 2).

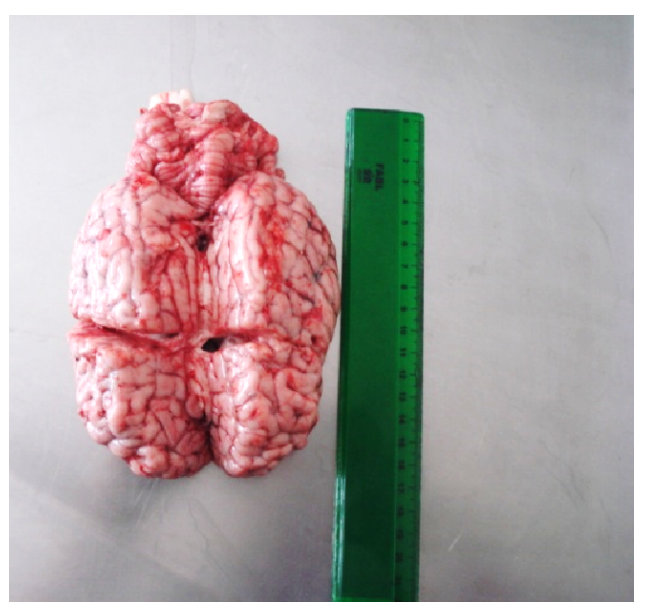

Fig. 2. Congestion, haemorrhages and friable consistency of the brain.

Samples from brain, liver, spleen, kidney, intestine, lung, stomach, heart and lymph node were collected and fixed with $10 \%$ neutral buffered formalin for $48 \mathrm{~h}$. The brain was fixed for $48 \mathrm{~h}$, after which was sliced at $\sim 5 \mathrm{~mm}$ thickness and fixed for another $48 \mathrm{~h}$ in fresh formalin, and the following areas were obtained: cortex, brain stem, cerebellum and medulla ob- longata. The tissues were sectioned and stained with haematoxylin and eosin (H\&E). Microscopically, histopathology showed a reduction of staining affinity (Fig. 3). Vaccuolation in both white and gray matter of cerebrum was obvious. Severe gliosis, haemorrhage and diffuse oedema were also seen. Cerebral cortex necrosis was mild but leukomalacia was severe. Although no gross lesions were apparent, histopathologic changes in the liver were recognisable and consisted of mild fatty degeneration, hepatocyte vacuolation and necrosis. Slight periportal fibrosis was also noted.

Samples from the suspected feed source were collected, cultured for fungi and submitted to the laboratory for mycotoxin evaluation. Pure Fusarium proliferatum culture was isolated from the feed sample. Determination of fumonisins by the specified ELISA revealed a concentration of $13.2 \mathrm{ppm}(13,200 \mu \mathrm{g} / \mathrm{kg})$ of fumonisins.

Acute central neurological disease in horses can be caused by several viruses (e.g. West Nile virus, equine herpes virus1 and other encephalitides viruses), protozoa (e.g. Sarcocystis neurona), bacteria (e.g. Listeria spp.), trauma and toxic substances (e.g. fumonisins and yellow starthistle, Centaurea solstitialis, intoxication).

Fumonisins are structurally related to sphingosine, the major long chain base backbone of cellular sphingolipids. They are competitive inhibitors of sphinganine and sphingosine $\mathrm{N}$-acyltransferase (also known as ceramide synthase), key enzymes in the de novo sphingolipid biosynthetic pathway. Sphingolipids are located in cellular membranes, lipoproteins especially low-density lipoproteins), and other lipid-rich structures. Complex sphingolipids are critical for the maintenance of 


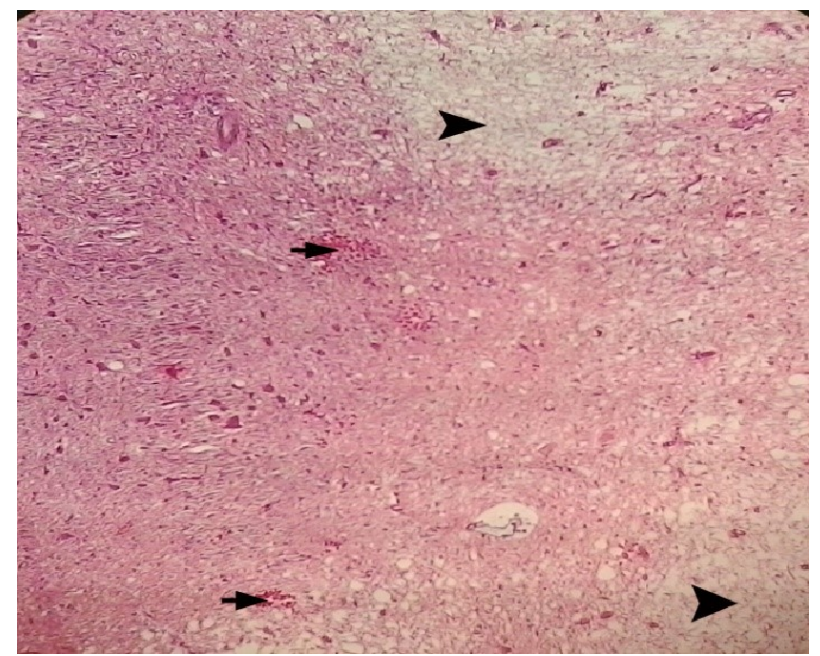

Fig. 3. Foci of necrosis (arrowhead) with no recognisable structure consistent with liquefactive necrosis in cerebral white matter. Arrows show perivascular congestion and oedema in the margin of normal and necrotic tissue structure $(\mathrm{H} \& \mathrm{E}, \times 160)$.

membrane structure, particularly microdomains such as caveolae. This enzyme inhibition by fumonisin produces a disruption of sphingolipid metabolism resulting in increased sphinganine and sphingosine along with a decrease in complex sphingolipids in the serum and tissues of animals. Therefore, high serum sphinganine, sphingosine, and sphinganine-to-sphingosine ratio suggest acute fumonisin toxicosis, but these assays are only available at research laboratories (Huggins, 2006; Smith, 2007).

Several reports have considered ELEM and hepatotoxicity to be two separate syndromes associated with fumonisin toxicity in horses. Field outbreaks of ELEM have been reported in Argentina, Brazil, China, Egypt, Greece, Iran, New Caledonia, South Africa, and especially the United States (Del Fava et al., 2010). However, it appears more likely these are not true "distinct" syndromes but related to the concentration of fumonisin in the feed, the duration of toxin consumption, and the tolerance of the individual horse to fumonisin (Smith, 2007) In the study by Del Fava (2010) clinical and pathological findings of leukoencephalomalacia in equids with neurological signs were described. Seven cases were reported during a two year course in Sao Paolo, Brazil. Giannitti et al. (2011) reported a case of ELEM due to fumonisins B1 and B2 in Argentina, the authors confirmed their diagnosis by detection of toxic concentrations of FB1 and FB2 $(17741 \mu \mathrm{g} / \mathrm{kg}$ or $17.7 \mathrm{ppm})$ in the feed supplement that the animals were eating. In Iran, Raoofi et al. (2003) reported an outbreak of ELEM in which 14 horses and 3 donkeys became affected by ingestion of mouldy alfalfa hay. ELEM were confirmed based on the clinical and pathological evidence and culture of $F$. moniliforme. In the case presented, the history and clinical sings were suggestive of ELEM. The pathological lesions, liquefactive necrosis of white matter, on cerebral cortex were characteristic of the disease. Finally the diagnosis 
was confirmed by detection of toxic concentrations of FB1 (13.2 ppm) in the commercially-prepared feedlot concentrates that the animal was eating. This value is well over the amount of fumonisin considered toxic for horse. Most naturally occurring cases of ELEM have been seen in animals eating feedstuff with fumonisins concentrations above $10 \mathrm{ppm}$ (Ross et al., 1991). Horses appear to be the most susceptible domestic animal species and can show signs when exposed to toxin concentrations as low as 5 to $10 \mathrm{ppm}$ (Smith, 2007). Regulatory guidance issued by the U.S. Food and Drug Administration calls for fumonisin $(\mathrm{B} 1+\mathrm{B} 2+\mathrm{B} 3)$ concentrations of no more than $5 \mathrm{ppm}$ in horse feeds (Smith, 2007).

In conclusion, the authors believe that the disease may be under-reported and it should be considered as an important differential diagnosis in horses that develop acute neurologic signs especially when are consuming feedstuffs containing corn.

\section{REFERENCES}

Del Fava, C., M. C. C. S. H. Lara, E. M. C. Villalobos, A. F. Nassar, A. D. Cabral, C. S. Torreli, M. S. Cunha \& E. M. Cunha, 2010. Leucoencefalomalacia (LEME) em equídeos no estado de São Paulo, Brasil: achados anatomopatológicos. Brazilian Journal of Veterinary Research and Animal Science, 47, 488494.

Giannitti, F., S. Sain Diab, P. A. Maria, M. Barrandeguy, C. Larrere, J. Ortega \& F. A. Uzal, 2011. Equine leukoencephalomalacia (ELEM) due to fumonisins B1 and B2 in Argentina. Pesquisa Veterinária Brasileira, 31, 407-412.
Huggins, A. J. \& J. R. Snyder, 2006. The nervous system. In: The Equine Manual, $2^{\text {nd }}$ edn, ed C. N. Hahn, Saunders, London, UK. pp. 1121-1122.

Raoofi, A., S. H. Mardjanmehr, A. R. Khosravi, G. A. Kojouri, S. Lotfollahzaheh, S. Nekoie \& M. Jafarian, 2003. Equine Leukoencephalomalacia in Iran. Journal of Equine Veterinary Science, 23, 469-470.

Reed, S., W. Bayly \& D. Sellon, 2004. Toxicologic problems. In: Equine Internal Medicine, $3^{\text {rd }}$ edn, D. G. Schmitz, Elsevier, St. Louis. pp. 1475-1477.

Robinson, N. E. \& K. A. Sprayberry, 2003. Changes in mentation, seizures, and narcolepsy. In: Current Therapy in Equine Medicine, $5^{\text {th }}$ edn, P. Washington, Saunders, St. Louis, Missouri. pp. 765-766.

Ross, P. F., L. G. Rice, J. C. Reagor, G. D. Osweiler, T. M. Wilson, H. A. Nelson, D. L.Owens, R. D. Plattner, K. A. Harlin, J. L. Richard, B. M. Colvin \& M. I. Banton, 1991. Fumonisin B1 concentrations in feeds from 45 confirmed equine leukoencephalomalacia cases. Journal of Veterinary Diagnostic Investigation, 3, 238-241.

Smith, G. W., 2007. Fumonisins. In: Veterinary Toxicology: Basic and Clinical Principles, $2^{\text {nd }}$ edn, ed R. C. Gupta, Academic Press. pp. 1205-1218.

Paper received 24.12.2017; accepted for publication 16.03.2018

\section{Correspondence:}

Parham Mottaghian

Faculty of Veterinary Medicine, University of Tehran, Tehran, Iran e-mail:p.mottaghian@ut.ac.ir 